\title{
A Straightforward Synthesis of N-Substituted Ureas from Primary Amides
}

\author{
Nathalie Saraiva Rosa ${ }^{a}$ \\ Thomas Glachet ${ }^{a}$ (D) \\ Quentin Ibert ${ }^{\mathrm{a}}$ (D) \\ Jean-François Lohier ${ }^{a}$ \\ Xavier Franck ${ }^{\mathbf{b}}$ (D) \\ Vincent Reboul*a (D) \\ a Normandie Univ, ENSICAEN, UNICAEN, CNRS, LCMT, \\ 14000 Caen, France \\ vincent.reboul@ensicaen.fr \\ b Normandie Univ, CNRS, UNIROUEN, INSA Rouen, COBRA, \\ 76000 Rouen, France
}

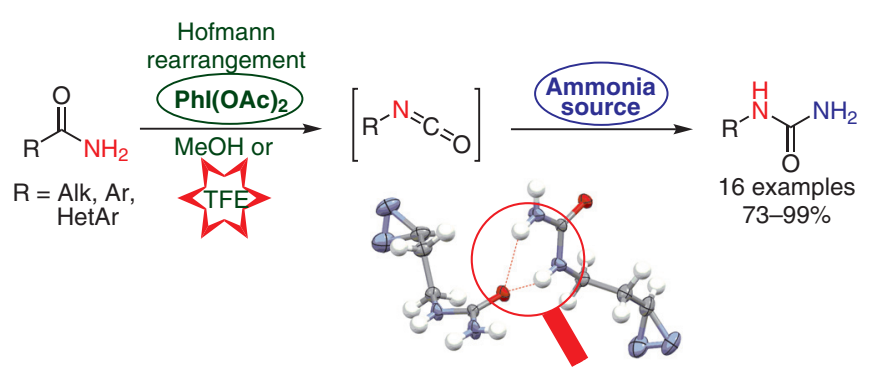

Received: 13.02 .2020

Accepted after revision: 06.04.2020

Published online: 27.04 .2020

DOI: 10.1055/s-0040-1707103; Art ID: ss-2020-z0087-op

Abstract A direct and convenient method for the preparation of $\mathrm{N}$-substituted ureas is achieved by treating primary amides with phenyliodine diacetate (PIDA) in the presence of an ammonia source $\left(\mathrm{NH}_{3}\right.$ or ammonium carbamate) in $\mathrm{MeOH}$. The use of 2,2,2-trifluoroethanol (TFE) as the solvent increases the electrophilicity of the hypervalent iodine species and allows the synthesis of electron-poor carboxamides. This transformation involves a nucleophilic addition of ammonia on the isocyanate intermediate generated in situ by a Hofmann rearrangement of the starting amide.

Key words amides, ureas, hypervalent iodine, Hofmann rearrangement, isocyanates

Due to their interesting physicochemical and biological properties, urea-containing molecules are increasingly used in various research fields such as medicinal chemistry, ${ }^{1}$ agrochemistry $^{2}$ and petrochemistry. ${ }^{3}$ In particular, the extensive utilization of such moieties in drug discovery as bioisosteres of peptide bonds arises from their structural, biological and electronic similarities. ${ }^{4}$

This key structural motif (Figure 1) can be found in natural products such as the amino acid citrulline (1) and allantoin (2), in marketed drugs such as cetrorelix (a decapeptide for in vitro fertilization) $)^{5}$ and degarelix (treatment of prostate cancer), ${ }^{6}$ and in several molecules with different biological activities such as anticancer $(\mathbf{3}$, a checkpoint kinase inhibitor $)^{7}$ anti-obesity (4), ${ }^{8}$ antiviral $(\mathbf{5})^{9}$ and antibacterial $(\mathbf{6}) .{ }^{10}$ In addition, primary ureas are not only useful in medicinal chemistry but can also be employed as versatile building blocks for further transformations. ${ }^{11}$

Since the first synthesis of urea from ammonium cyanate by Wöhler in 1828 , historically considered as the birth of organic chemistry, ${ }^{12}$ researchers have developed a myri-
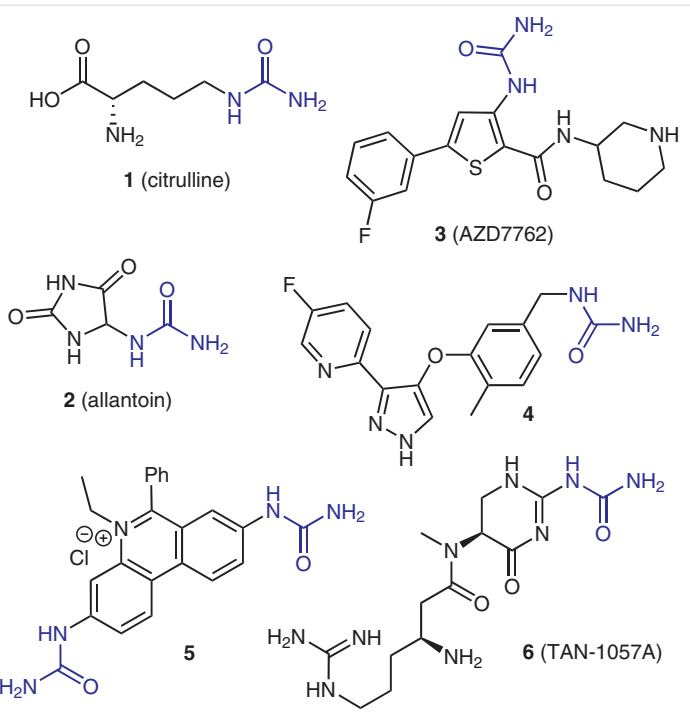

Figure 1 Biologically active compounds containing a primary urea moiety

ad of synthetic methods in order to prepare them. One of the most common method implies the reaction between ammonia and isocyanates, albeit with safety concerns. ${ }^{13}$ Consequently, isocyanates are usually formed in situ thanks tively from primary carboxamides, acyl azides and bis-acylated hydroxylamines. Interestingly, hypervalent iodine species appeared to be suitable oxidizing reagents for the Hofmann rearrangement, since they are mild, powerful, and are able to react with amide substrates without added base. Under various conditions, amines, ${ }^{15}$ carbamates ${ }^{16}$ and symmetric 1,3 -disubstituted ureas $^{17}$ can be prepared (Scheme 1). to Hofmann, Curtius or Lossen rearrangements, ${ }^{14}$ respec- 
Herein, we report a straightforward synthesis of primary alkyl- and arylureas (Scheme 1 ) by addition of ammonia to in situ generated isocyanates, proceeding via the Hofmann rearrangement of primary amides induced by phenyliodine diacetate (PIDA).

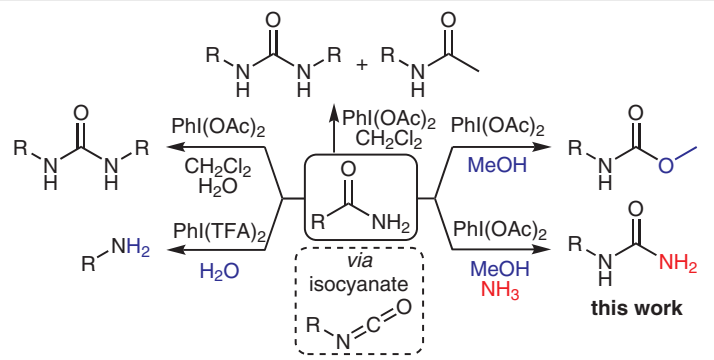

Scheme 1 Reactions of carboxamides with hypervalent iodine species

Numerous methods have been developed for the synthesis of primary ureas (Scheme 2) using different precursors. Examples include carboxylic acids, ${ }^{18}$ phenyl carbamate, ${ }^{19}$ anilines in the presence of urea, ${ }^{20}$ aryl chlorides by Pd-catalyzed cross-couplings with benzylurea followed by in situ hydrogenolysis, ${ }^{21}$ arylcyanamides by hydration reactions (with or without $\mathrm{HCO}_{2} \mathrm{H}$ ), ${ }^{22}$ amines by nucleophilic addition to potassium isocyanate, ${ }^{23}$ nitriles via the Tiemann rearrangement, ${ }^{24}$ or arenes by $\mathrm{CH}$ amination. ${ }^{25}$

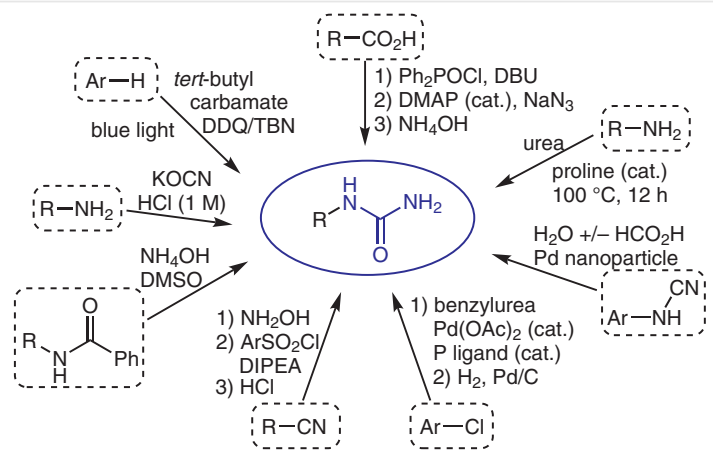

Scheme 2 Literature methods for the preparation of primary ureas

Originally, the unexpected formation of an N-substituted urea was observed during the investigation of the onepot synthesis of $3 H$-diazirines from $\alpha$-amino acids, ${ }^{26}$ using PIDA and a methanolic solution of ammonia. Under these conditions, L-glutamine afforded a crystalline compound $\mathbf{7}$, $\mathrm{X}$-ray analysis of which $^{27}$ revealed that the amide moiety was converted into the corresponding primary urea (Scheme 3).

Since the formation of methyl carbamate $\mathbf{8}^{19}$ was not observed, despite the presence of methanol in the reaction medium, ${ }^{28}$ we assumed that the isocyanate intermediate, formed in situ via a Hofmann-like rearrangement, ${ }^{29}$ was trapped by ammonia which is more nucleophilic (Scheme 4).

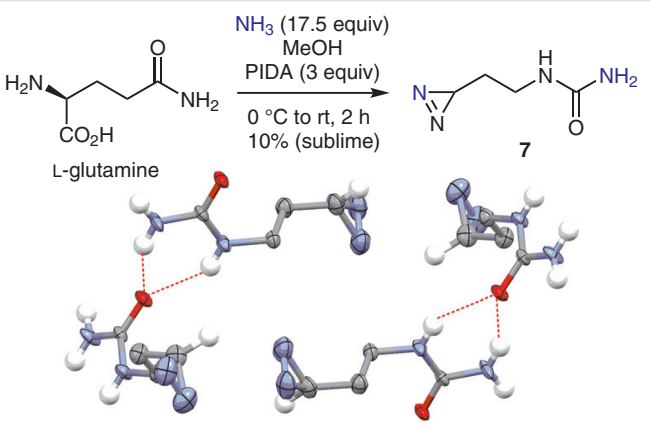

Scheme 3 Formation of diazirine $\mathbf{7}$ (from L-glutamine) and its X-ray crystal structure (packing with $\mathrm{H}$-bonding)

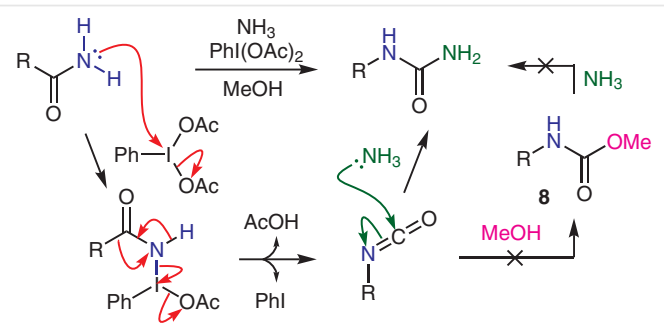

Scheme 4 Proposed mechanism

Based on this encouraging result, optimization of the reaction conditions was carried out using 4-methoxybenzamide as a model substrate (Table 1). Using the same amounts of methanolic ammonia and PIDA, the reaction performed at $0{ }^{\circ} \mathrm{C}$ afforded the desired urea 9a in a better yield than at room temperature (entries 1 and 2 ). Increasing the amount of PIDA to 2 equivalents improved the yield of the reaction, however, higher amounts were not beneficial (entries 3 and 4). Finally, 17.5 equivalents of methanolic ammonia were required to obtain the urea in a quantitative yield (entries 5-7).

Table 1 Optimization of the Reaction Conditions for the Formation of 9a

\begin{tabular}{lllll}
\hline & & & \\
\hline Entry & PIDA (equiv) & $\mathrm{NH}_{3}$ (equiv) & $\mathrm{Temp}$ & Isolated yield (\%) \\
\hline 1 & 1 & 5.0 & $\mathrm{rt}$ & 47 \\
2 & 1 & 5.0 & $0{ }^{\circ} \mathrm{C}$ to rt & 51 \\
3 & 2 & 5.0 & $0{ }^{\circ} \mathrm{C}$ to rt & 70 \\
4 & 3 & 5.0 & $0{ }^{\circ} \mathrm{C}$ to rt & 50 \\
5 & 2 & 10.0 & $0{ }^{\circ} \mathrm{C}$ to rt & 80 \\
6 & 2 & 15.0 & $0{ }^{\circ} \mathrm{C}$ to rt & 85 \\
7 & 2 & 17.5 & $0{ }^{\circ} \mathrm{C}$ to rt & $>99$ \\
\hline
\end{tabular}


With optimized conditions in hand (conditions A), different alkyl-, aryl- and heteroaryl-amides were converted into the corresponding primary ureas 9a-r (Scheme 5).

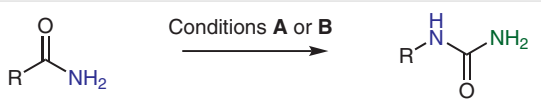

Conditions A: $\mathrm{NH}_{3}$ (17.5 equiv), PIDA (2 equiv), $\mathrm{MeOH}, 0{ }^{\circ} \mathrm{C}$ to rt, $2 \mathrm{~h}$ Conditions B: AC (1.5 equiv), PIDA (2 equiv), TFE, $0^{\circ} \mathrm{C}$ to rt, $9 \mathrm{~h}$ then $A C$ (1 equiv), PIDA (1 equiv), rt, $12 \mathrm{~h}$

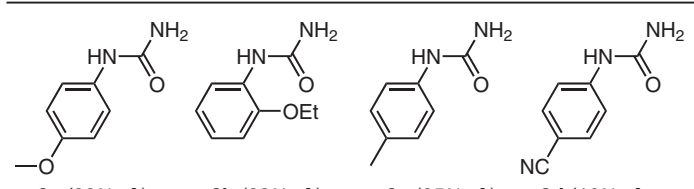

9a $(99 \%, \mathbf{A}) \quad 9 b(99 \%, \mathbf{A}) \quad$ 9c $(95 \%, \mathbf{A}) \quad 9 d(16 \%, \mathbf{A}$
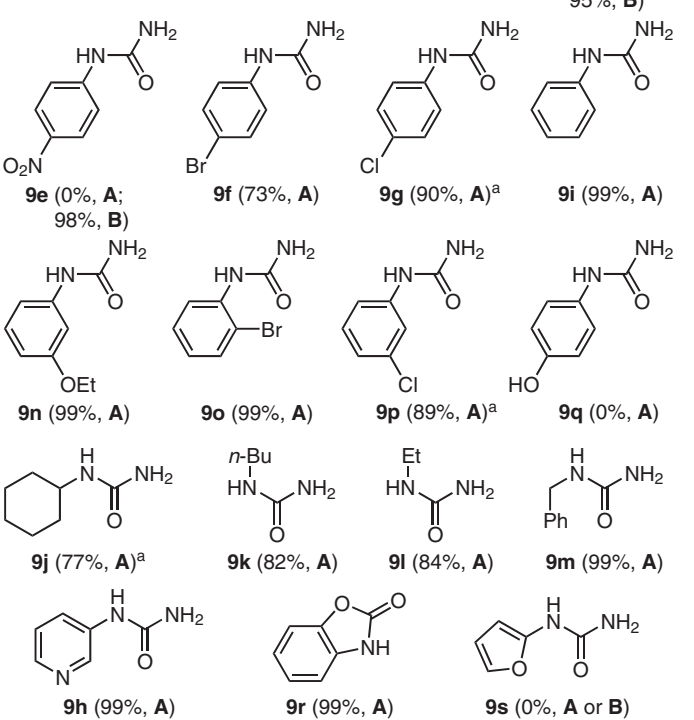

Scheme 5 Scope of the reaction. ${ }^{\text {a }}$ An additional amount of PIDA/NH was added and the reaction time was extended to $48 \mathrm{~h}$.

Reactions with aromatic amides bearing electrondonating (9a-c,n) or deactivating groups (9f,0) afforded the corresponding ureas in good to excellent yields, as well as pyridyl- (9h), phenyl- (9i) and benzylureas $(\mathbf{9 m})$. Although aliphatic amides were fully converted into the expected ureas (9k,1), the loss of small amounts of the products during purification was observed due to their volatility and consequently impacted their isolated yields. Furthermore, the reaction time was extended to 48 hours to achieve the synthesis of compounds $\mathbf{9 g}, \mathbf{9 j}$ and $\mathbf{9 p}$ in high yields. On the other hand, the presence of an electron-withdrawing group on the aromatic amide seemed to lower the reaction yield of this transformation since 4-cyanophenylurea 9d was obtained in only $16 \%$ yield and 4 -nitrophenylurea $9 e$ could not be prepared using this procedure. ${ }^{30}$ However, it was possible to increase the electrophilicity of PIDA by using a highly polar and strong hydrogen-bond donating solvent, such as 2,2,2-trifluoroethanol (TFE). ${ }^{31}$ Moreover, we reasoned that using a slow ammonia release source, such as ammonium carbamate $(A C)$, would improve the yield as we had already shown in the sulfoximination reaction of sulfides. ${ }^{32}$ Hence, it was found that performing the reaction using conditions B (Scheme 5) allowed the synthesis of compounds 9d and 9e in $95 \%$ and $98 \%$ yields, respectively. In contrast, a substrate with an aromatic ring bearing a 4-OH group failed to give the desired urea $\mathbf{9 q},{ }^{33}$ whereas that with a 2-OH group gave the cyclic carbamate $9 \mathbf{r}$ isolated in quantitative yield. However, 2-furamide did not react under both sets of conditions.

In conclusion, an easy, mild and affordable method to convert primary carboxamides into $\mathrm{N}$-substituted ureas has been developed by utilizing a PIDA-induced Hofmann rearrangement followed by addition of ammonia. The reactivity of the hypervalent reagent was increased by using TFE as the solvent and $\mathrm{AC}$ as the ammonia source, allowing the synthesis of aromatic ureas bearing electron-withdrawing groups. This reaction allows access to a wide range of alkyl-, aryl- and heteroarylureas which could potentially possess interesting biological activities.

Column chromatography was performed on Merck silica gel (230-400 mesh). Thin-layer chromatography was performed using Merck precoated, aluminium-backed silica gel plates. Melting points were determined using a Gallen-Kamp melting point apparatus. IR spectra were recorded on a PerkinElmer ATR-spectrum one spectrometer. ${ }^{1} \mathrm{H}$ NMR spectra were recorded at $500 \mathrm{MHz}$ or $600 \mathrm{MHz}$ and ${ }^{13} \mathrm{C}$ NMR spectra were recorded at $125 \mathrm{MHz}$ or $150 \mathrm{MHz}$ on Bruker Avance III 500 and Avance 600 Neo spectrometers. High-resolution mass spectrometry (HRMS) was performed using a Xevo G2-XS QTof Waters mass spectrometer equipped with an electrospray ion source (ESI) operated in positive ion mode.

\section{N-Substituted Ureas; General Procedure A}

(Diacetoxyiodo)benzene ( $1.0 \mathrm{mmol}, 2.0$ equiv) was added in one portion to a stirred solution of the amide $(0.5 \mathrm{mmol}, 1.0$ equiv) in $\mathrm{NH}_{3} / \mathrm{MeOH}\left(7 \mathrm{M}, 1.25 \mathrm{~mL}, 17.5\right.$ equiv) at $0{ }^{\circ} \mathrm{C}$ under argon. After 30 min at $0{ }^{\circ} \mathrm{C}$, the reaction mixture was allowed to reach room temperature and was left to stir for $90 \mathrm{~min}$. After completion (monitored by TLC and ${ }^{1} \mathrm{H}$ NMR), the reaction mixture was concentrated under reduced pressure and the crude product was purified by flash chromatography on silica gel.

\section{N-Substituted Ureas; General procedure B}

(Diacetoxyiodo)benzene ( $1.0 \mathrm{mmol}, 2.0$ equiv) was added in one portion to a stirred solution of the amide ( $0.5 \mathrm{mmol}, 1.0$ equiv) and ammonium carbamate (AC) $(0.75 \mathrm{mmol}, 1.5$ equiv) in trifluoroethanol $(1.25 \mathrm{~mL})$ at $0{ }^{\circ} \mathrm{C}$ under argon. After $30 \mathrm{~min}$ at $0{ }^{\circ} \mathrm{C}$, the reaction mixture was allowed to reach room temperature and was left to stir for 9 h. Additional amounts of PIDA and AC (1.0 equiv each) were added at $\mathrm{rt}$ and the reaction mixture was stirred for $12 \mathrm{~h}$ at rt. After concentration of the reaction mixture under reduced pressure, the crude product was purified by flash chromatography on silica gel. 


\section{1-(4-Methoxyphenyl)urea (9a)}

Prepared according to General Procedure A using 4-methoxybenzamide ( $75.6 \mathrm{mg}, 0.5 \mathrm{mmol}$ ) and purified by flash chromatography on silica gel $\left(\mathrm{CH}_{2} \mathrm{Cl}_{2} / \mathrm{MeOH}, 95: 5\right)$ to afford urea 9a (83.1 mg, 99\%) as a brown solid.

Mp $163{ }^{\circ} \mathrm{C} ; R_{f}=0.2\left(\mathrm{CH}_{2} \mathrm{Cl}_{2} / \mathrm{MeOH}, 95: 5\right)$.

IR (ATR): 3469, 3301, 1643, 1542, 1215, 1037, 822, $561 \mathrm{~cm}^{-1}$.

${ }^{1} \mathrm{H}$ NMR (600 MHz, DMSO- $d_{6}$ ): $\delta=8.30(\mathrm{~s}, 1 \mathrm{H}, \mathrm{NH}), 7.28(\mathrm{~d}, J=9.0 \mathrm{~Hz}$, $\left.2 \mathrm{H}, \mathrm{CH}_{\mathrm{Ar}}\right), 6.80\left(\mathrm{~d}, \mathrm{~J}=9.0 \mathrm{~Hz}, 2 \mathrm{H}, \mathrm{CH}_{\mathrm{Ar}}\right), 5.71\left(\mathrm{~s}, 2 \mathrm{H}, \mathrm{NH}_{2}\right), 3.68(\mathrm{~s}, 3 \mathrm{H}$, $\left.\mathrm{OCH}_{3}\right)$.

${ }^{13} \mathrm{C}$ NMR $\left(150 \mathrm{MHz}, \mathrm{DMSO}-d_{6}\right): \delta=156.2(\mathrm{CO}), 153.9\left(\mathrm{C}_{\mathrm{Ar}}\right), 133.7\left(\mathrm{C}_{\mathrm{Ar}}\right)$, $119.4\left(\mathrm{C}_{\mathrm{Ar}}\right), 113.8\left(\mathrm{C}_{\mathrm{Ar}}\right), 55.1\left(\mathrm{OCH}_{3}\right)$.

HRMS (ESI-QTOF): $m / z[M+H]^{+}$calcd for $\mathrm{C}_{8} \mathrm{H}_{11} \mathrm{~N}_{2} \mathrm{O}_{2}: 167.0821$; found: 167.0821 .

\section{1-(2-Ethoxyphenyl)urea (9b)}

Prepared according to General Procedure A using 2-ethoxybenzamide ( $82.6 \mathrm{mg}, 0.5 \mathrm{mmol}$ ) and purified by flash chromatography on silica gel $\left(\mathrm{CH}_{2} \mathrm{Cl}_{2} / \mathrm{MeOH}, 95: 5\right)$ to afford urea $9 \mathbf{b}(90.1 \mathrm{mg}, 99 \%)$ as a brown solid.

Mp $115^{\circ} \mathrm{C} ; R_{f}=0.3\left(\mathrm{CH}_{2} \mathrm{Cl}_{2} / \mathrm{MeOH}, 95: 5\right)$.

IR (ATR): 3484, 3328, 3198, 1661, 1525, 1450, 1249, 1045, $746 \mathrm{~cm}^{-1}$.

${ }^{1} \mathrm{H}$ NMR $\left(600 \mathrm{MHz}\right.$, DMSO- $\left.d_{6}\right): \delta=8.07\left(\mathrm{dd}, J=7.8,1.8 \mathrm{~Hz}, 1 \mathrm{H}, \mathrm{CH}_{A r}\right)$, $7.75(\mathrm{~s}, 1 \mathrm{H}, \mathrm{NH}), 6.93\left(\mathrm{dd}, J=7.8,1.8 \mathrm{~Hz}, 1 \mathrm{H}, \mathrm{CH}_{A r}\right), 6.79-6.85(\mathrm{~m}, 2 \mathrm{H}$, $\mathrm{CH}_{\mathrm{Ar}}$ ), 6.24 (br s, $2 \mathrm{H}, \mathrm{NH}_{2}$ ), $4.08\left(\mathrm{q}, J=7.0 \mathrm{~Hz}, 2 \mathrm{H}, \mathrm{OCH}_{2} \mathrm{CH}_{3}\right), 1.38$ (t, $J=7.0 \mathrm{~Hz}, 3 \mathrm{H}, \mathrm{OCH}_{2} \mathrm{CH}_{3}$ ).

${ }^{13} \mathrm{C}$ NMR $\left(150 \mathrm{MHz}\right.$, DMSO- $\left.d_{6}\right): \delta=156.0(\mathrm{CO}), 146.5\left(\mathrm{C}_{\mathrm{Ar}}\right), 129.7\left(\mathrm{C}_{\mathrm{Ar}}\right)$, $121.0\left(\mathrm{C}_{\mathrm{Ar}}\right), 120.4\left(\mathrm{C}_{\mathrm{Ar}}\right), 118.3\left(\mathrm{C}_{\mathrm{Ar}}\right), 111.6\left(\mathrm{C}_{\mathrm{Ar}}\right), 63.8\left(\mathrm{OCH}_{2} \mathrm{CH}_{3}\right), 14.7$ $\left(\mathrm{OCH}_{2} \mathrm{CH}_{3}\right)$

HRMS (ESI-QTOF): $m / z[M+H]^{+}$calcd for $\mathrm{C}_{9} \mathrm{H}_{13} \mathrm{~N}_{2} \mathrm{O}_{2}$ : 181.0977; found: 181.0977 .

\section{1-(p-Tolyl)urea (9c)}

Prepared according to General Procedure A using p-toluamide (67.6 $\mathrm{mg}, 0.5 \mathrm{mmol}$ ) and purified by flash chromatography on silica gel $\left(\mathrm{CH}_{2} \mathrm{Cl}_{2} / \mathrm{MeOH}, 95: 5\right)$ to afford urea $9 \mathbf{c}(71.3 \mathrm{mg}$, 95\%) as a white solid. Mp $180{ }^{\circ} \mathrm{C} ; R_{f}=0.4\left(\mathrm{CH}_{2} \mathrm{Cl}_{2} / \mathrm{MeOH}, 95: 5\right)$.

IR (ATR): 3422, 3306, 1650, 1589, 1546, 1354, 811, 550, $501 \mathrm{~cm}^{-1}$.

${ }^{1} \mathrm{H}$ NMR $\left(500 \mathrm{MHz}\right.$, DMSO- $\left.d_{6}\right): \delta=8.37(\mathrm{~s}, 1 \mathrm{H}, \mathrm{NH}), 7.26(\mathrm{~d}, J=8.1 \mathrm{~Hz}$, $\left.2 \mathrm{H}, \mathrm{CH}_{A r}\right), 7.01\left(\mathrm{~d}, J=8.1 \mathrm{~Hz}, 2 \mathrm{H}, \mathrm{CH}_{A r}\right), 5.75\left(\mathrm{~s}, 2 \mathrm{H}, \mathrm{NH}_{2}\right), 2.20(\mathrm{~s}, 3 \mathrm{H}$, $\mathrm{CH}_{3}$ ).

${ }^{13} \mathrm{C}$ NMR (125 MHz, DMSO- $\left.d_{6}\right): \delta=156.0(C O), 138.0\left(C_{\mathrm{Ar}}\right), 129.7\left(\mathrm{C}_{\mathrm{Ar}}\right)$, $129.0\left(\mathrm{C}_{\mathrm{Ar}}\right), 117.8\left(\mathrm{C}_{\mathrm{Ar}}\right), 20.3\left(\mathrm{CH}_{3}\right)$.

HRMS (ESI-QTOF): $m / z[\mathrm{M}+\mathrm{H}]^{+}$calcd for $\mathrm{C}_{8} \mathrm{H}_{11} \mathrm{~N}_{2} \mathrm{O}: 151.0871$; found: 151.0873 .

\section{1-(4-Cyanophenyl)urea (9d)}

Prepared according to General Procedure B using 4-cyanobenzamide (73.1 $\mathrm{mg}, 0.5 \mathrm{mmol}$ ) and purified by flash chromatography on silica gel $\left(\mathrm{CH}_{2} \mathrm{Cl}_{2} / \mathrm{EtOH}, 97: 3\right)$ to afford urea 9d (76.5 mg, 95\%) as a white solid

Mp $220{ }^{\circ} \mathrm{C} ; R_{f}=0.2\left(\mathrm{CH}_{2} \mathrm{Cl}_{2} / \mathrm{EtOH}, 97: 3\right)$.

IR (ATR): 3484, 3379, 2219, 1678, 1587, 1538, 1361, 835, $510 \mathrm{~cm}^{-1}$

${ }^{1} \mathrm{H}$ NMR $\left(500 \mathrm{MHz}\right.$, DMSO- $\left.d_{6}\right): \delta=9.07(\mathrm{~s}, 1 \mathrm{H}, \mathrm{NH}), 7.65(\mathrm{~d}, J=8.5 \mathrm{~Hz}$, $\left.2 \mathrm{H}, \mathrm{CH}_{\mathrm{Ar}}\right), 7.57\left(\mathrm{~d}, J=8.5 \mathrm{~Hz}, 2 \mathrm{H}, \mathrm{CH}_{\mathrm{Ar}}\right), 6.12\left(\mathrm{~s}, 2 \mathrm{H}, \mathrm{NH}_{2}\right)$.
${ }^{13} \mathrm{C}$ NMR $\left(125 \mathrm{MHz}\right.$, DMSO- $\left.d_{6}\right): \delta=155.5(\mathrm{CO}), 145.1\left(\mathrm{C}_{\mathrm{Ar}}\right), 133.1\left(\mathrm{C}_{\mathrm{Ar}}\right)$, $119.5(\mathrm{CN}), 117.5\left(\mathrm{C}_{\mathrm{Ar}}\right), 102.4\left(\mathrm{C}_{\mathrm{Ar}}\right)$.

HRMS (ESI-QTOF): $m / z$ [M $+\mathrm{H}]^{+}$calcd for $\mathrm{C}_{8} \mathrm{H}_{8} \mathrm{~N}_{3} \mathrm{O}: 162.0667$; found: 162.0667 .

\section{1-(4-Nitrophenyl)urea (9e)}

Prepared according to General Procedure B using 4-nitrobenzamide ( $83.1 \mathrm{mg}, 0.5 \mathrm{mmol}$ ) and purified by flash chromatography on silica gel $\left(\mathrm{CH}_{2} \mathrm{Cl}_{2} / \mathrm{EtOH}, 97: 3\right)$ to afford urea $9 e(88.7 \mathrm{mg}, 98 \%)$ as a white solid.

Mp $225^{\circ} \mathrm{C} ; R_{f}=0.1\left(\mathrm{CH}_{2} \mathrm{Cl}_{2} / \mathrm{EtOH}, 97: 3\right)$.

IR (ATR): 3486, 3378, 1687, 1547, 1483, 1316, 1095, 854, $692 \mathrm{~cm}^{-1}$.

${ }^{1} \mathrm{H}$ NMR $\left(500 \mathrm{MHz}\right.$, DMSO- $\left.d_{6}\right): \delta=9.31$ (br s, $\left.1 \mathrm{H}, \mathrm{NH}\right), 8.13$ (d, $J=9.1$ $\mathrm{Hz}, 2 \mathrm{H}, \mathrm{CH}_{A r}$ ), 7.63 (d, J = 9.1 Hz, $2 \mathrm{H}, \mathrm{CH}_{A r}$ ), 6.22 (br s, $2 \mathrm{H}, \mathrm{NH}_{2}$ ).

${ }^{13} \mathrm{C}$ NMR $\left(125 \mathrm{MHz}\right.$, DMSO- $\left.d_{6}\right): \delta=155.3(\mathrm{CO}), 147.3\left(\mathrm{C}_{\mathrm{Ar}}\right), 140.4\left(\mathrm{C}_{\mathrm{Ar}}\right)$, $125.1\left(\mathrm{C}_{\mathrm{Ar}}\right), 116.9\left(\mathrm{C}_{\mathrm{Ar}}\right)$.

HRMS (ESI-QTOF): $m / z$ [M $+\mathrm{H}]^{+}$calcd for $\mathrm{C}_{7} \mathrm{H}_{8} \mathrm{~N}_{3} \mathrm{O}_{3}$ : 182.0566; found: 182.0567 .

\section{1-(4-Bromophenyl)urea (9f)}

Prepared according to General Procedure A using 4-bromobenzamide ( $100.0 \mathrm{mg}, 0.5 \mathrm{mmol}$ ) and purified by flash chromatography on silica gel $\left(\mathrm{CH}_{2} \mathrm{Cl}_{2} / \mathrm{MeOH}, 95: 5\right)$ to afford urea $\mathbf{9 f}(78.0 \mathrm{mg}, 73 \%)$ as a white solid.

$\mathrm{Mp} 226^{\circ} \mathrm{C} ; R_{f}=0.3\left(\mathrm{CH}_{2} \mathrm{Cl}_{2} / \mathrm{MeOH}, 95: 5\right)$.

IR (ATR): 3417, 3306, 3213, 1651, 1582, 1544, 1486, 1353, 815, 586 $\mathrm{cm}^{-1}$.

${ }^{1} \mathrm{H}$ NMR $\left(500 \mathrm{MHz}\right.$, DMSO- $\left.d_{6}\right): \delta=8.67(\mathrm{~s}, 1 \mathrm{H}, \mathrm{NH}), 7.37\left(\mathrm{~s}, 4 \mathrm{H}, \mathrm{CH}_{A r}\right)$, $5.91\left(\mathrm{~s}, 2 \mathrm{H}, \mathrm{NH}_{2}\right)$.

${ }^{13} \mathrm{C}$ NMR $\left(125 \mathrm{MHz}\right.$, DMSO- $\left.d_{6}\right): \delta=155.8(\mathrm{CO}), 140.0\left(\mathrm{C}_{\mathrm{Ar}}\right), 131.3\left(\mathrm{C}_{\mathrm{Ar}}\right)$, $119.6\left(\mathrm{C}_{\mathrm{Ar}}\right), 112.3\left(\mathrm{C}_{\mathrm{Ar}}\right)$.

HRMS (ESI-QTOF): $m / z[\mathrm{M}+\mathrm{H}]^{+}$calcd for $\mathrm{C}_{7} \mathrm{H}_{8} \mathrm{~N}_{2} \mathrm{OBr}$ : 214.9820; found: 214.9832 .

\section{1-(4-Chlorophenyl)urea (9g)}

Prepared according to General Procedure A using 4-chlorobenzamide ( $77.8 \mathrm{mg}, 0.5 \mathrm{mmol})$. Additional amounts of PIDA $(0.25 \mathrm{mmol})$ and $\mathrm{NH}_{3}$ in $\mathrm{MeOH}(7 \mathrm{M}, 0.3 \mathrm{~mL})$ were added after $16 \mathrm{~h}$ and the mixture was left stirring for an additional $24 \mathrm{~h}$. The mixture was concentrated under reduced pressure and the residue was purified by flash chromatography on silica gel $\left(\mathrm{CH}_{2} \mathrm{Cl}_{2} / \mathrm{EtOH}, 95: 5\right)$ to afford urea $\mathbf{9 g}(76.8$ mg, 90\%) as a white solid.

Mp $209^{\circ} \mathrm{C} ; R_{f}=0.3\left(\mathrm{CH}_{2} \mathrm{Cl}_{2} / \mathrm{MeOH}, 95: 5\right)$.

IR (ATR): 3419, 3311, 1651, 1545, 1490, 1090, 820, 585, $489 \mathrm{~cm}^{-1}$.

${ }^{1} \mathrm{H}$ NMR $\left(500 \mathrm{MHz}\right.$, DMSO- $\left.d_{6}\right): \delta=8.66(\mathrm{~s}, 1 \mathrm{H}, \mathrm{NH}), 7.42(\mathrm{~d}, J=8.5 \mathrm{~Hz}$, $\left.2 \mathrm{H}, \mathrm{CH}_{A r}\right), 7.24\left(\mathrm{~d}, J=8.5 \mathrm{~Hz}, 2 \mathrm{H}, \mathrm{CH}_{A r}\right), 5.90\left(\mathrm{~s}, 2 \mathrm{H}, \mathrm{NH}_{2}\right)$.

${ }^{13} \mathrm{C}$ NMR $\left(125 \mathrm{MHz}, \mathrm{DMSO}-d_{6}\right): \delta=155.8(\mathrm{CO}), 139.6\left(\mathrm{C}_{\mathrm{Ar}}\right), 128.4\left(\mathrm{C}_{\mathrm{Ar}}\right)$, $124.5\left(C_{\mathrm{Ar}}\right), 119.2\left(\mathrm{C}_{\mathrm{Ar}}\right)$.

HRMS (ESI-QTOF): $m / z[\mathrm{M}+\mathrm{H}]^{+}$calcd for $\mathrm{C}_{7} \mathrm{H}_{8} \mathrm{~N}_{2} \mathrm{OCl}$ : 171.0325; found: 171.0328

\section{1-(Pyridin-3-yl)urea (9h)}

Prepared according to General Procedure A using nicotinamide (66.2 $\mathrm{mg}, 0.5 \mathrm{mmol}$ ) and purified by flash chromatography on silica gel $\left(\mathrm{CH}_{2} \mathrm{Cl}_{2} / \mathrm{MeOH}, 90: 10+\mathrm{a}\right.$ few drops of $\left.\mathrm{NH}_{3}\right)$ to afford urea $\mathbf{9 h}(68.6$ $\mathrm{mg}, 99 \%$ ) as a yellow solid. 
Mp $199{ }^{\circ} \mathrm{C} ; R_{f}=0.3\left(\mathrm{CH}_{2} \mathrm{Cl}_{2} / \mathrm{MeOH}, 90: 10+\right.$ a few drops of $\left.\mathrm{NH}_{3}\right)$. IR (ATR): 3374, 3198, 1672, 1550, 1485, 1356, 1302, $571 \mathrm{~cm}^{-1}$. ${ }^{1} \mathrm{H}$ NMR $\left(600 \mathrm{MHz}\right.$, DMSO- $\left.d_{6}\right): \delta=8.73(\mathrm{~s}, 1 \mathrm{H}, \mathrm{NH}), 8.51(\mathrm{~d}, J=2.4 \mathrm{~Hz}$, $\left.1 \mathrm{H}, \mathrm{CH}_{\mathrm{Ar}}\right), 8.10\left(\mathrm{dd}, J=4.6,1.4 \mathrm{~Hz}, 1 \mathrm{H}, \mathrm{CH}_{\mathrm{Ar}}\right), 7.89$ (ddd, $J=8.3,2.6,1.5$ $\mathrm{Hz}, 1 \mathrm{H}, \mathrm{CH}_{A r}$ ), 2.23 (ddd, J = 8.3, 4.6, $0.5 \mathrm{~Hz}, 1 \mathrm{H}, \mathrm{CH}_{A r}$ ), 6.01 (br s, $2 \mathrm{H}$, $\mathrm{NH}_{2}$ )

${ }^{13} \mathrm{C}$ NMR $\left(150 \mathrm{MHz}, \mathrm{DMSO}-d_{6}\right): \delta=156.0(\mathrm{CO}), 142.1\left(\mathrm{C}_{\mathrm{Ar}}\right), 139.6\left(\mathrm{C}_{\mathrm{Ar}}\right)$, 137.2 $\left(\mathrm{C}_{\mathrm{Ar}}\right), 124.5\left(\mathrm{C}_{\mathrm{Ar}}\right), 123.5\left(\mathrm{C}_{\mathrm{Ar}}\right)$.

HRMS (ESI-QTOF): $m / z$ [M + H] $]^{+}$calcd for $\mathrm{C}_{6} \mathrm{H}_{8} \mathrm{~N}_{3} \mathrm{O}$ : 138.0667; found: 138.0670 .

\section{1-Phenylurea (9i)}

Prepared according to General Procedure A using benzamide (60.6 $\mathrm{mg}, 0.5 \mathrm{mmol}$ ) and purified by flash chromatography on silica gel $\left(\mathrm{CH}_{2} \mathrm{Cl}_{2} / \mathrm{EtOH}, 97: 3\right)$ to afford urea $\mathbf{9 i}(67.8 \mathrm{mg}$, $>99 \%)$ as a beige solid. Mp $149{ }^{\circ} \mathrm{C} ; R_{f}=0.2\left(\mathrm{CH}_{2} \mathrm{Cl}_{2} / \mathrm{EtOH}, 97: 3\right)$.

IR (ATR): 3420, 3311, 3214, 1651, 1590, 1548, 1353, 750, 694, $584 \mathrm{~cm}^{-1}$. ${ }^{1} \mathrm{H}$ NMR $\left(500 \mathrm{MHz}\right.$, DMSO- $\left.d_{6}\right): \delta=8.49(\mathrm{~s}, 1 \mathrm{H}, \mathrm{NH}), 7.38(\mathrm{~d}, J=7.7 \mathrm{~Hz}$, $\left.2 \mathrm{H}, \mathrm{CH}_{A r}\right), 7.20\left(\mathrm{t}, J=7.7 \mathrm{~Hz}, 2 \mathrm{H}, \mathrm{CH}_{A r}\right), 6.88\left(\mathrm{t}, J=7.7 \mathrm{~Hz}, 1 \mathrm{H}, \mathrm{CH}_{A r}\right)$, $5.82\left(\mathrm{~s}, 2 \mathrm{H}, \mathrm{NH}_{2}\right)$.

${ }^{13} \mathrm{C}$ NMR (125 MHz, DMSO- $\left.d_{6}\right): \delta=156.0(C O), 140.6\left(\mathrm{C}_{\mathrm{Ar}}\right), 128.6\left(\mathrm{C}_{\mathrm{Ar}}\right)$, $121.0\left(\mathrm{C}_{\mathrm{Ar}}\right), 117.7\left(\mathrm{C}_{\mathrm{Ar}}\right)$.

HRMS (ESI-QTOF): $m / z$ [M $+\mathrm{H}]^{+}$calcd for $\mathrm{C}_{7} \mathrm{H}_{9} \mathrm{~N}_{2} \mathrm{O}: 137.0715$; found: 137.0713.

\section{1-Cyclohexylurea (9j)}

Prepared according to General Procedure A using cyclohexanecarboxamide ( $63.6 \mathrm{mg}, 0.5 \mathrm{mmol})$. Additional amounts of PIDA $(0.15 \mathrm{mmol})$ and $\mathrm{NH}_{3}$ in $\mathrm{MeOH}(7 \mathrm{M}, 0.18 \mathrm{~mL}$ ) were added after $16 \mathrm{~h}$ and the mixture was left stirring for an additional $24 \mathrm{~h}$. The mixture was concentrated under reduced pressure and the residue was purified by flash chromatography on silica gel $\left(\mathrm{CH}_{2} \mathrm{Cl}_{2} / \mathrm{MeOH}, 95: 5\right)$ to afford urea 9j $(55.0 \mathrm{mg}, 77 \%)$ as a white solid.

Mp $195{ }^{\circ} \mathrm{C} ; R_{f}=0.2\left(\mathrm{CH}_{2} \mathrm{Cl}_{2} / \mathrm{MeOH}, 95: 5\right)$.

IR (ATR): 3328, 3196, 2928, 2852, 1649, 1544, 1351, 1157, $609 \mathrm{~cm}^{-1}$.

${ }^{1} \mathrm{H}$ NMR (500 MHz, DMSO- $\left.d_{6}\right): \delta=8.81(\mathrm{~d}, J=8.0 \mathrm{~Hz}, 1 \mathrm{H}, \mathrm{NH}), 5.28(\mathrm{br}$ $\left.\mathrm{s}, 2 \mathrm{H}, \mathrm{NH}_{2}\right), 3.26-3.32(\mathrm{~m}, 1 \mathrm{H}, \mathrm{CH}), 1.71-1.73\left(\mathrm{~m}, 2 \mathrm{H}, \mathrm{CH}_{2}\right), 1.61-$ $1.64\left(\mathrm{~m}, 2 \mathrm{H}, \mathrm{CH}_{2}\right), 1.50-1.52(\mathrm{~m}, 1 \mathrm{H}, \mathrm{CH}), 1.20-1.28\left(\mathrm{~m}, 2 \mathrm{H}, \mathrm{CH}_{2}\right)$, 1.10-1.20 (m, $1 \mathrm{H}, \mathrm{CHH}), 1.01-1.10(\mathrm{~m}, 2 \mathrm{H}, \mathrm{CH})$.

${ }^{13} \mathrm{C}$ NMR $\left(125 \mathrm{MHz}\right.$, DMSO- $\left.d_{6}\right): \delta=157.9(\mathrm{CO}), 47.6\left(\mathrm{C}_{\mathrm{Ar}}\right), 33.3\left(\mathrm{C}_{\mathrm{Ar}}\right)$, $25.3\left(\mathrm{C}_{\mathrm{Ar}}\right), 24.5\left(\mathrm{C}_{\mathrm{Ar}}\right)$.

HRMS (ESI-QTOF): $m / z[\mathrm{M}+\mathrm{H}]^{+}$calcd for $\mathrm{C}_{7} \mathrm{H}_{15} \mathrm{~N}_{2} \mathrm{O}$ : 143.1184 ; found: 143.1185 .

\section{1-Butylurea (9k)}

Prepared according to General Procedure A using valeramide (50.6 $\mathrm{mg} ; 0.5 \mathrm{mmol}$ ) and purified by flash chromatography on silica gel $\left(\mathrm{CH}_{2} \mathrm{Cl}_{2} / \mathrm{EtOH}, 9: 1\right)$ to afford urea $9 \mathbf{k}(47.5 \mathrm{mg}, 82 \%)$ as colorless needles.

Mp $98^{\circ} \mathrm{C} ; R_{f}=0.3\left(\mathrm{CH}_{2} \mathrm{Cl}_{2} / \mathrm{EtOH}, 90: 10\right)$.

IR (ATR): 3411, 3348, 3208, 2968, 2932, 1554, 1339, 1157, $533 \mathrm{~cm}^{-1}$. ${ }^{1} \mathrm{H}$ NMR $\left(500 \mathrm{MHz}\right.$, DMSO- $\left.d_{6}\right): \delta=5.87(\mathrm{~s}, 1 \mathrm{H}, \mathrm{NH}), 5.34\left(\mathrm{~s}, 2 \mathrm{H}, \mathrm{NH}_{2}\right)$, $2.93\left(\mathrm{q}, J=6.5 \mathrm{~Hz}, 2 \mathrm{H}, \quad \mathrm{NHCH}_{2} \mathrm{CH}_{2}\right), 1.23-1.34(\mathrm{~m}, 4 \mathrm{H}$, $\left.\mathrm{H}_{3} \mathrm{CCH}_{2} \mathrm{CH}_{2} \mathrm{CH}_{2}\right), 0.86$ (t, $J=7.2 \mathrm{~Hz}, 3 \mathrm{H}, \mathrm{CH}_{3}$ ).

${ }^{13} \mathrm{C}$ NMR (125 MHz, DMSO- $\left.d_{6}\right): \delta=158.7(\mathrm{CO}), 38.8\left(\mathrm{CH}_{2}\right), 32.1\left(\mathrm{CH}_{2}\right)$, $19.5\left(\mathrm{CH}_{2}\right), 13.7\left(\mathrm{CH}_{3}\right)$.
HRMS (ESI-QTOF): $m / z[\mathrm{M}+\mathrm{Na}]^{+}$calcd for $\mathrm{C}_{7} \mathrm{H}_{12} \mathrm{~N}_{2} \mathrm{ONa}$ : 139.0847; found: 139.0844.

\section{1-Ethylurea (91)}

Prepared according to General Procedure A using propionamide (73.1 $\mathrm{mg}, 1.0 \mathrm{mmol}$ ) and purified by flash chromatography on silica gel $\left(\mathrm{CH}_{2} \mathrm{Cl}_{2} / \mathrm{EtOH}, 9: 1\right)$ to afford urea 91 (74.3 $\left.\mathrm{mg}, 84 \%\right)$ as colorless needles.

Mp $83{ }^{\circ} \mathrm{C} ; R_{f}=0.2\left(\mathrm{CH}_{2} \mathrm{Cl}_{2} / \mathrm{EtOH}, 90: 10\right)$.

IR (ATR): 3350, 3207, 2931, 1548, 1340, 1157, $536 \mathrm{~cm}^{-1}$.

${ }^{1} \mathrm{H}$ NMR $\left(500 \mathrm{MHz}\right.$, DMSO- $\left.d_{6}\right): \delta=5.86(\mathrm{~s}, 1 \mathrm{H}, \mathrm{NH}), 5.36\left(\mathrm{~s}, 2 \mathrm{H}, \mathrm{NH}_{2}\right)$, 2.96 (quin, $J=7.0 \mathrm{~Hz}, 2 \mathrm{H}, \mathrm{CH}_{2}$ ), $0.96\left(\mathrm{t}, J=7.2 \mathrm{~Hz}, 3 \mathrm{H}, \mathrm{CH}_{3}\right.$ ).

${ }^{13} \mathrm{C}$ NMR (125 MHz, DMSO- $\left.d_{6}\right): \delta=158.6(\mathrm{CO}), 34.0\left(\mathrm{CH}_{2}\right), 15.7\left(\mathrm{CH}_{3}\right)$. HRMS (ESI-QTOF): $m / z$ [M $+\mathrm{H}]^{+}$calcd for $\mathrm{C}_{3} \mathrm{H}_{9} \mathrm{~N}_{2} \mathrm{O}$ : 89.0715; found: 89.0713.

\section{1-Benzylurea (9m)}

Prepared according to General Procedure A using 2-phenylacetamide (67.6 $\mathrm{mg}, 0.5 \mathrm{mmol}$ ) and purified by flash chromatography on silica gel $\left(\mathrm{CH}_{2} \mathrm{Cl}_{2} / \mathrm{MeOH}, 95: 5\right)$ to afford urea $9 \mathrm{~m}(75.1 \mathrm{mg}, 86 \%)$ as a white solid.

Mp $150{ }^{\circ} \mathrm{C} ; R_{\mathrm{f}}=0.3\left(\mathrm{CH}_{2} \mathrm{Cl}_{2} / \mathrm{MeOH}, 95: 5\right)$.

IR (ATR): 3428, 3328, 1647, 1598, 1557, 695, 583, $546 \mathrm{~cm}^{-1}$.

${ }^{1} \mathrm{H}$ NMR $\left(500 \mathrm{MHz}\right.$, DMSO- $\left.d_{6}\right): \delta=7.20-7.32\left(\mathrm{~m}, 5 \mathrm{H}, \mathrm{CH}_{\mathrm{Ar}}\right), 6.40(\mathrm{t}$, $J=6.0 \mathrm{~Hz}, 1 \mathrm{H}, \mathrm{NH}$ ), 5.52 (br s, $\left.2 \mathrm{H}, \mathrm{NH}_{2}\right), 4.17\left(\mathrm{~d}, J=6.0 \mathrm{~Hz}, 2 \mathrm{H}, \mathrm{CH}_{2}\right.$ ).

${ }^{13} \mathrm{C}$ NMR $\left(125 \mathrm{MHz}\right.$, DMSO- $\left.d_{6}\right): \delta=158.7(\mathrm{CO}), 140.9\left(\mathrm{C}_{\mathrm{Ar}}\right), 128.2\left(\mathrm{C}_{\mathrm{Ar}}\right)$, $127.0\left(\mathrm{C}_{\mathrm{Ar}}\right), 126.5\left(\mathrm{C}_{\mathrm{Ar}}\right), 42.8\left(\mathrm{CH}_{2}\right)$.

HRMS (ESI-QTOF): $m / z$ [M $+\mathrm{H}]^{+}$calcd for $\mathrm{C}_{8} \mathrm{H}_{11} \mathrm{~N}_{2} \mathrm{O}: 151.0871$; found: 151.0870

\section{1-(3-Ethoxyphenyl)urea (9n)}

Prepared according to General Procedure A using 3-ethoxybenzamide ( $82.6 \mathrm{mg}, 0.5 \mathrm{mmol}$ ) and purified by flash chromatography on silica gel $\left(\mathrm{CH}_{2} \mathrm{Cl}_{2} / \mathrm{MeOH}, 95: 5\right)$ to afford urea 9 n (90.0 mg, 99\%) as a brown solid.

Mp $112{ }^{\circ} \mathrm{C} ; R_{f}=0.3\left(\mathrm{CH}_{2} \mathrm{Cl}_{2} / \mathrm{MeOH}, 95: 5\right)$.

IR (ATR): 3434, 3307, 3208, 1652, 1531, 1191, 1044, 766, $593 \mathrm{~cm}^{-1}$.

${ }^{1} \mathrm{H}$ NMR $\left(500 \mathrm{MHz}\right.$, DMSO- $\left.d_{6}\right): \delta=8.48(\mathrm{~s}, 1 \mathrm{H}, \mathrm{NH}), 7.06-7.12(\mathrm{~m}, 2 \mathrm{H}$, $\left.\mathrm{CH}_{A r}\right), 6.83\left(\mathrm{~d}, J=8.0 \mathrm{~Hz}, 1 \mathrm{H}, \mathrm{CH}_{A r}\right), 6.44\left(\mathrm{~d}, J=8.0 \mathrm{~Hz}, 1 \mathrm{H}, \mathrm{CH}_{A r}\right), 5.82$ $\left(\mathrm{s}, 2 \mathrm{H}, \mathrm{NH}_{2}\right), 3.94\left(\mathrm{q}, J=6.8 \mathrm{~Hz}, 2 \mathrm{H}, \mathrm{CH}_{2}\right), 1.30\left(\mathrm{t}, J=6.8 \mathrm{~Hz}, 3 \mathrm{H}, \mathrm{CH}_{3}\right)$. ${ }^{13} \mathrm{C}$ NMR $\left(125 \mathrm{MHz}\right.$, DMSO- $\left.d_{6}\right): \delta=158.9(\mathrm{CO}), 155.9\left(\mathrm{C}_{\mathrm{Ar}}\right), 141.8\left(\mathrm{C}_{\mathrm{Ar}}\right)$, $129.3\left(\mathrm{C}_{\mathrm{Ar}}\right), 110.0\left(\mathrm{C}_{\mathrm{Ar}}\right), 106.9\left(\mathrm{C}_{\mathrm{Ar}}\right), 104.0\left(\mathrm{C}_{\mathrm{Ar}}\right), 62.7\left(\mathrm{CH}_{2}\right), 14.7\left(\mathrm{CH}_{3}\right)$. HRMS (ESI-QTOF): $m / z[\mathrm{M}+\mathrm{H}]^{+}$calcd for $\mathrm{C}_{9} \mathrm{H}_{13} \mathrm{~N}_{2} \mathrm{O}_{2}$ : 181.0977; found: 181.0977 .

\section{1-(2-Bromophenyl)urea (9o)}

Prepared according to General Procedure A using 2-bromobenzamide (100.0 mg, $0.5 \mathrm{mmol}$ ) and purified by flash chromatography on silica gel $\left(\mathrm{CH}_{2} \mathrm{Cl}_{2} / \mathrm{MeOH}, 95: 5\right)$ to afford urea $90(107.5 \mathrm{mg}$, 99\%) as an offwhite solid.

Mp $206{ }^{\circ} \mathrm{C} ; R_{f}=0.3\left(\mathrm{CH}_{2} \mathrm{Cl}_{2} / \mathrm{MeOH}, 95: 5\right)$.

IR (ATR): 3419, 3287, 3196, 1651, 1516, 1474, 1354, 755, $539 \mathrm{~cm}^{-1}$.

${ }^{1} \mathrm{H}$ NMR (500 MHz, DMSO- $d_{6}$ ): $\delta=8.00\left(\mathrm{~d}, J=8.2 \mathrm{~Hz}, 1 \mathrm{H}, \mathrm{CH}_{A r}\right), 7.85$ $(\mathrm{s}, 1 \mathrm{H}, \mathrm{NH}), 7.54\left(\mathrm{~d}, J=7.8 \mathrm{~Hz}, 1 \mathrm{H}, \mathrm{CH}_{A r}\right), 7.26\left(\mathrm{t}, J=7.8 \mathrm{~Hz}, 1 \mathrm{H}, \mathrm{CH}_{A r}\right)$, $7.89\left(\mathrm{t}, J=7.8 \mathrm{~Hz}, 1 \mathrm{H}, \mathrm{CH}_{A r}\right), 6.40\left(\mathrm{~s}, 2 \mathrm{H}, \mathrm{NH}_{2}\right)$. 
${ }^{13} \mathrm{C}$ NMR $\left(125 \mathrm{MHz}\right.$, DMSO- $\left.d_{6}\right): \delta=155.6(C O), 137.9\left(\mathrm{C}_{\mathrm{Ar}}\right), 132.3\left(\mathrm{C}_{\mathrm{Ar}}\right)$, $127.9\left(\mathrm{C}_{\mathrm{Ar}}\right), 123.3\left(\mathrm{C}_{\mathrm{Ar}}\right), 121.9\left(\mathrm{C}_{\mathrm{Ar}}\right), 112.4\left(\mathrm{C}_{\mathrm{Ar}}\right)$.

HRMS (ESI-QTOF): $m / z$ [M + Na] ${ }^{+}$calcd for $\mathrm{C}_{7} \mathrm{H}_{8} \mathrm{~N}_{2} \mathrm{OBrNa}$ : 236.9639; found: 236.9640 .

\section{1-(3-Chlorophenyl)urea (9p)}

Prepared according to General Procedure A using 3-chlorobenzamide ( $77.8 \mathrm{mg}, 0.5 \mathrm{mmol}$ ). Additional amounts of PIDA $(0.5 \mathrm{mmol})$ and $\mathrm{NH}_{3}$ in $\mathrm{MeOH}(7 \mathrm{M}, 0.60 \mathrm{~mL}$ ) were added after $4 \mathrm{~h}$ and the mixture was left to stir for an additional $24 \mathrm{~h}$. The mixture was concentrated under reduced pressure and the residue was purified by flash chromatography on silica gel $\left(\mathrm{CH}_{2} \mathrm{Cl}_{2} / \mathrm{MeOH}, 95: 5\right)$ to afford urea $\mathbf{9 p}(76.2 \mathrm{mg}, 89 \%)$ as a pale brown solid.

Mp $143{ }^{\circ} \mathrm{C} ; R_{f}=0.2\left(\mathrm{CH}_{2} \mathrm{Cl}_{2} / \mathrm{MeOH}, 95: 5\right)$.

${ }^{1} \mathrm{H}$ NMR $\left(500 \mathrm{MHz}\right.$, DMSO- $\left.d_{6}\right): \delta=8.73(\mathrm{~s}, 1 \mathrm{H}, \mathrm{NH}), 7.69(\mathrm{t}, J=2.0 \mathrm{~Hz}$, $\left.1 \mathrm{H}, \mathrm{CH}_{A r}\right), 7.22\left(\mathrm{t}, J=8.2 \mathrm{~Hz}, 1 \mathrm{H}, \mathrm{CH}_{A r}\right), 7.16(\mathrm{ddd}, J=8.2,1.9,1.0 \mathrm{~Hz}, 1$ $\mathrm{H}, \mathrm{CH}_{A r}$ ), 6.92 (ddd, $J=7.8,2.0,1.0 \mathrm{~Hz}, 1 \mathrm{H}, \mathrm{CH}_{\mathrm{Ar}}$ ), $5.96\left(\mathrm{~s}, 2 \mathrm{H}, \mathrm{NH}_{2}\right.$ ).

${ }^{13} \mathrm{C}$ NMR $\left(125 \mathrm{MHz}\right.$, DMSO- $\left.d_{6}\right): \delta=155.8(\mathrm{CO}), 142.2\left(\mathrm{C}_{\mathrm{Ar}}\right), 133.1\left(\mathrm{C}_{\mathrm{Ar}}\right)$, $130.2\left(\mathrm{C}_{\mathrm{Ar}}\right), 120.6\left(\mathrm{C}_{\mathrm{Ar}}\right), 117.0\left(\mathrm{C}_{\mathrm{Ar}}\right), 116.0\left(\mathrm{C}_{\mathrm{Ar}}\right)$.

HRMS (ESI-QTOF): $m / z\left[M+\mathrm{H}^{+}\right.$calcd for $\mathrm{C}_{7} \mathrm{H}_{8} \mathrm{~N}_{2} \mathrm{OCl}$ : 171.0325; found: 171.0325 .

\section{2(3H)-Benzoxazolone (9r)}

Prepared according to General Procedure A using salicylamide (68.6 $\mathrm{mg}, 0.5 \mathrm{mmol}$ ) and purified by flash chromatography on silica gel $\left(\mathrm{CH}_{2} \mathrm{Cl}_{2} / \mathrm{MeOH}, 95: 5\right)$ to afford urea $9 \mathbf{r}(67 \mathrm{mg}, 99 \%)$ as a brown solid.

Mp $140{ }^{\circ} \mathrm{C} ; R_{f}=0.4\left(\mathrm{CH}_{2} \mathrm{Cl}_{2} / \mathrm{MeOH}, 95: 5\right)$.

IR (ATR): 3201, 1732, 1478, 1253, 937, $686 \mathrm{~cm}^{-1}$.

${ }^{1} \mathrm{H}$ NMR (500 MHz, DMSO- $\left.d_{6}\right): \delta=11.6(\mathrm{~s}, 1 \mathrm{H}, \mathrm{NH}), 7.27$ (d, $J=8.0 \mathrm{~Hz}$, $\left.1 \mathrm{H}, \mathrm{CH}_{A r}\right), 7.12-7.16\left(\mathrm{~m}, 1 \mathrm{H}, \mathrm{CH}_{A r}\right), 7.06-7.09\left(\mathrm{~m}, 2 \mathrm{H}, \mathrm{CH}_{A r}\right)$.

${ }^{13} \mathrm{C}$ NMR $\left(125 \mathrm{MHz}\right.$, DMSO- $\left.d_{6}\right): \delta=154.4(\mathrm{CO}), 143.3\left(\mathrm{C}_{\mathrm{Ar}}\right), 130.4\left(\mathrm{C}_{\mathrm{Ar}}\right)$, $123.8\left(\mathrm{C}_{\mathrm{Ar}}\right), 121.8\left(\mathrm{C}_{\mathrm{Ar}}\right), 109.8\left(\mathrm{C}_{\mathrm{Ar}}\right), 109.5\left(\mathrm{C}_{\mathrm{Ar}}\right)$.

HRMS (ESI-QTOF): $m / z$ [M + H] $]^{+}$calcd for $\mathrm{C}_{7} \mathrm{H}_{6} \mathrm{NO}_{2}$ : 136.0399; found: 136.0401.

\section{Funding Information}

The authors thank the Centre National de la Recherche Scientifique (CNRS), Normandie Université (RIN ChemImaging), Labex SynOrg (Grant No. ANR-11-LABX-0029), the Conseil Régional de Normandie and the Fonds Européen de Développement Régional (FEDER) for financial support.

\section{Acknowledgment}

We gratefully acknowledged K. Jarsalé for HRMS analyses, and $\mathrm{H}$. El Slibani and R. Legay for NMR studies carried out at Caen.

\section{Supporting Information}

Supporting information for this article is available online at https://doi.org/10.1055/s-0040-1707103.

\section{References}

(1) (a) Ghosh, A. K.; Brindisi, M. J. Med. Chem. 2020, 63, 2751. (b) Jagtap, A. D.; Kondekar, N. B.; Sadani, A. A.; Chern, J. W. Curr. Med. Chem. 2017, 24, 622. (c) Cioffi, C. L.; Dobri, N.; Freeman, E. E.; Conlon, M. P.; Chen, P.; Stafford, D. G.; Schwarz, D. M. C.; Golden, K. C.; Zhu, L.; Kitchen, D. B.; Barnes, K. D.; Racz, B.; Qin, Q.; Michelotti, E.; Cywin, C. L.; Martin, W. H.; Pearson, P. G.; Johnson, G.; Petrukhin, K. J. Med. Chem. 2014, 57, 7731. (d) Belfrage, A. K.; Gising, J.; Svensson, F.; Åkerblom, E.; Sköld, C.; Sandström, A. Eur. J. Med. Chem. 2015, 978. (e) Nowotarski, S. L.; Pachaiyappan, B.; Holshouser, S. L.; Kutz, C. J.; Li, Y.; Huang, Y.; Sharma, S. K.; Casero, R. A. Jr.; Woster, P. M. Bioorg. Med. Chem. 2015, 23, 1601. (f) Darvesh, S.; Pottie, I. R.; Darvesh, K. V.; McDonald, R. S.; Walsh, R.; Conrad, S.; Penwell, A.; Mataija, D.; Martin, E. Bioorg. Med. Chem. 2010, 18, 2232. (g) Manickam, M.; Pillaiyar, T.; Boggu, P.; Venkateswararao, E.; Jalani, H. B.; Kim, N.-D.; Lee, S. K.; Jeon, J. S.; Kim, S. K.; Jung, S.-H. Eur. J. Med. Chem. 2016, 117, 113. (h) Nepali, K.; Sharma, S.; Sharma, M.; Bedi, P. M. S.; Dhar, K. L. Eur. J. Med. Chem. 2014, 77, 422. (i) Gong, H.; Yang, M.; Xiao, Y. Z.; Doweyko, A. M.; Cunningham, M.; Wang, J.; Habte, S.; Holloway, D.; Burke, C.; Shuster, D.; Gao, L.; Carman, J.; Somerville, J. E.; Nadler, S. G.; Salter-Cid, L.; Barrish, J. C.; Weinstein, D. S. Bioorg. Med. Chem. Lett. 2014, 24 , 3268. (j) Li, H.-Q.; Lv, P. C.; Yan, T.; Zhu, H.-L. Anti-Cancer Agents Med. Chem. 2009, 9, 471.

(2) (a) Zakrzewski, J.; Krawczyk, M. Heteroat. Chem. 2006, 17, 393. (b) Wada, Y.; Kamada, Y.; Hanaki, K. US Patent 5833733, 1998. (c) Landes, M.; Sievernich, B.; Kibler, E.; Nuyken, W.; Walter, H.; Westphalen, K.-O.; Mayer, H.; Haden, E.; Mulder, C.; Schönhammer, A.; Hamprecht, G. US Patent 6054410, 2000. (d) Achgill, R. K.; Call, L. W. US Patent 4987233, 1991. (e) Arnold, W. R. US Patent 4165229, 1979.

(3) Klingstedt, F.; Arve, K.; Eränen, K.; Murzin, D. Y. Acc. Chem. Res. 2006, 39, 273.

(4) Volz, N.; Clayden, J. Angew. Chem. Int. Ed. 2011, 50, 12148.

(5) Pullagurla, M. R.; Rangisetty, J. B. US Patent 20190382447, 2019.

(6) Guryanov, I.; Orlandin, A.; Viola, A.; Biondi, B.; Badocco, D.; Formaggio, F.; Ricci, A.; Cabri, W. Org. Process Res. Dev. 2019, 23 , 2746.

(7) Oza, V.; Ashwell, S.; Almeida, L.; Brassil, P.; Breed, J.; Deng, C.; Gero, T.; Grondine, M.; Horn, C.; Ioannidis, S.; Liu, D.; Lyne, P.; Newcombe, N.; Pass, M.; Read, J.; Ready, S.; Rowsell, S.; Su, M.; Toader, D.; Vasbinder, M.; Yu, D.; Yu, Y.; Xue, Y.; Zabludoff, S.; Janetka, J. J. Med. Chem. 2012, 55, 5130.

(8) Cui, K.; Chen, J.; Wang, M. PCT Int. Appl WO2016201662, 2016.

(9) Luedtke, N. W.; Liu, Q.; Tor, Y. Bioorg. Med. Chem. 2003, 11, 5235.

(10) Katayama, N.; Fukusumi, S.; Funabashi, Y.; Iwahi, T.; Ono, H. J. Antibiot. 1993, 46, 606.

(11) (a) Lee, S.-H.; Yoshida, K.; Matsushita, H.; Clapham, B.; Koch, G.; Zimmermann, J.; Janda, K. D. J. Org. Chem. 2004, 69, 8829. (b) Lee, S.-H.; Clapham, B.; Koch, G.; Zimmermann, J.; Janda, K. D. Org. Lett. 2003, 5, 511. (c) Belfrage, A. K.; Gising, J.; Svensson, F.; Åkerblom, E.; Sköld, C.; Sandström, A. Eur. J. Org. Chem. 2015, 978. (d) Wu, J.; Xie, Y.; Chen, X.; Deng, G.-J. Adv. Synth. Catal. 2016, 358, 3206. (e) Rakesh, K. P.; Ramesha, A. B.; Shantharam, C. S.; Mantelingu, K.; Mallesha, N. RSC Adv. 2016, 6, 108315. (f) Rekunge, D. S.; Khatri, C. K.; Chaturbhuj, G. U. Tetrahedron Lett. 2017, 58, 4304.

(12) Wöhler, F. Ann. Phys. Chem. 1828, 88, 253.

(13) Browne, D. L.; O’Brien, M.; Koos, P.; Cranwell, P. B.; Polyzos, A.; Ley, S. V. Synlett 2012, 23, 1402. 
(14) Aubé, J.; Fehl, C.; Liu, R.; McLeod, M. C.; Motiwala, H. F. In Comprehensive Organic Synthesis II, Vol. 6; Elsevier: Amsterdam, 2014, 598 .

(15) Loudon, G. M.; Radhakrishna, A. S.; Almond, M. R.; Blodgett, J. K.; Boutin, R. H. J. Org. Chem. 1984, 49, 4272.

(16) Moriarty, R. M.; Chany, C. J. II.; Vaid, R. K.; Prakash, O.; Tuladhar, S. M. J. Org. Chem. 1993, 58, 2478.

(17) (a) Landsberg, U.; Kalesse, M. Synlett 2010, 1104. (b) Liu, P.; Wang, Z.; Hu, X. Eur. J. Org. Chem. 2012, 1994.

(18) Kumar, A.; Kumar, N.; Sharma, R.; Bhargava, G.; Mahajan, D. J. Org. Chem. 2019, 84, 11323.

(19) Thavonekham, B. Synthesis 1997, 1189.

(20) Nagarkar, A. G.; Telvekar, V. N. Lett. Org. Chem. 2017, 15, 926.

(21) Breitler, S.; Oldenhuis, N. J.; Fors, B. P.; Buchwald, S. L. Org. Lett. 2011, 13, 3262.

(22) Habibi, D.; Heydari, S.; Faraji, A.; Keypour, H.; Mahmoudabadi, M. Polyhedron 2018, 151, 520.

(23) (a) Sardarian, A. R.; Inaloo, I. D. RSC Adv. 2015, 5, 76626. (b) Tiwari, L.; Kumar, V.; Kumar, B.; Mahajan, D. RSC Adv. 2018, 8, 21585.

(24) Wang, C. H.; Hsieh, T. H.; Lin, C. C.; Yeh, W. H.; Lin, C. A.; Chien, T. C. Synlett 2015, 26, 1823.

(25) Das, S.; Natarajan, P.; König, B. Chem. Eur. J. 2017, 23, 18161.
(26) Glachet, T.; Marzag, H.; Saraiva Rosa, N.; Colell, J. F. P.; Zhang, G.; Warren, W. S.; Franck, X.; Theis, T.; Reboul, V. J. Am. Chem. Soc. 2019, 141, 13689.

(27) Single crystals suitable for X-ray crystallographic analysis were obtained by slow evaporation from an $\mathrm{Et}_{2} \mathrm{O}$ solution. CCDC 1896523 contains the supplementary crystallographic data for this paper. The data can be obtained free of charge from The Cambridge Crystallographic Data Centre via www.ccdc.cam.ac.uk/getstructures.

(28) No reaction occurred between (4-methoxyphenyl)methyl carbamate and ammonia in methanol at rt.

(29) Boutin, R. H.; Loudon, G. M. J. Org. Chem. 1984, 49, 4277.

(30) The same trend was observed with other methods: see references 18,20 and $23 \mathrm{~b}$.

(31) Chaabouni, S.; Lohier, J. F.; Barthelemy, A. L.; Glachet, T.; Anselmi, E.; Dagousset, G.; Diter, P.; Pégot, B.; Magnier, E.; Reboul, V. Chem. Eur.J. 2018, 24, 17006.

(32) (a) Lohier, J.-F.; Glachet, T.; Marzag, H.; Gaumont, A.-C.; Reboul, V. Chem. Commun. 2017, 53, 2064. (b) Glachet, T.; Franck, X.; Reboul, V. Synthesis 2019, 51, 971.

(33) Pouységu, L.; Deffieux, D.; Quideau, S. Tetrahedron 2010, 66, 2235. 